\title{
Using a new set of space-borne particle monitors to investigate solar-terrestrial relations
}

\author{
K. Tziotziou ${ }^{1}$, I. Sandberg ${ }^{1}$, A. Anastasiadis ${ }^{1}$, I. A. Daglis ${ }^{1}$, and P. Nieminen ${ }^{2}$ \\ 1 National Observatory of Athens, Institute for Space Applications and Remote Sensing, 15236 Palea Penteli, Greece \\ e-mail: [kostas; sandberg; anastasi; daglis]@space.noa.gr \\ 2 European Space Agency, ESTEC, Postbus 299, Noordwijk, 2200 AG, The Netherlands \\ e-mail: petteri.nieminen@esa.int
}

Received 19 July 2009 / Accepted 23 January 2010

ABSTRACT

\begin{abstract}
Context. We investigated the solar origin of Solar Particle Events (SPEs) which occurred during four time periods of the 23rd solar cycle with intense solar activity and were detected by the Standard Radiation Environment Monitor (SREM) onboard the INTEGRAL satellite. SREM is a second generation ESA particle radiation monitor, already flying onboard seven spacecraft.

Aims. The present study attempts to establish the association of recorded SPEs by INTEGRAL/SREM with their solar sources and hence evaluate the potential of SREM units as an alarm system for hazardous SPEs.

Methods. X-ray, optical and radio data of solar flares and coronal mass ejections (CMEs) that were observed by several space-based instruments during the aforementioned solar cycle were reduced and thoroughly analyzed to establish the corresponding solar origin of the selected SPEs.

Results. The analysis produced clear associations with X-class solar flares and respective CMEs for the events of the OctoberNovember 2003, January 2005 and December 2006 periods, while for the events that occurred during September 2005, associations with X-class flares are possible but not straightforward due to the complexity of the registered solar particle fluxes.

Conclusions. SREM proves to be a new valuable asset for a comprehensive study of SPEs and a useful alert instrument for explosive geoeffective solar events.
\end{abstract}

Key words. Sun: activity - Sun: coronal mass ejections - Sun: flares - Sun: particle emission

\section{Introduction}

Solar particle events (SPEs) are particle radiation events caused by the Sun, consisting primarily of protons but also electrons and other heavy ions with energies ranging from a few tens of $\mathrm{keV}$ to $\mathrm{GeV}$ (Reames 1999). Their frequency of occurrence varies, but it is not unusual to see several (ten to twenty) significant SPEs within a solar cycle. Although most of them are relatively shortlived, usually lasting for tens of hours with an exponential decrease in flux afterwards, they can reach high values of fluxes at their peak, thus posing severe hazards for space missions, satellites and sensitive ground-based instruments. The most powerful ones can produce significant Ground Level Enhancements (GLEs) of the particle radiation levels on the surface of the Earth. SPEs are registered by several space-based and ground-based particle radiation monitors.

The Standard Radiation Environment Monitor (SREM) (Bühler et al. 1996) is a second generation solid state-based detector, which measures both protons with energies above $10 \mathrm{MeV}$ and electrons with energies above $500 \mathrm{keV}$, continuously monitoring the particle radiation environment and thus providing valuable protection for the host spacecraft and its payload when particle radiation hazards are imminent. So far, seven units have been launched on-board satellites STRV1C, PROBA-1, INTEGRAL, ROSETTA, GIOVE-B and recently on HERSCHEL and PLANCK, while two additional units are planned to be launched on as yet to be determined missions.

The principal advantage of SREM compared to other particle radiation monitors is the existence of many identical SREM units in several different missions with diverse orbits, covering both the near-Earth environment (geospace) as well as the interplanetary space. Several puzzling and persistent questions about SPEs that relate to energy release processes on the Sun, the particle acceleration and the characteristics of the (radiation) environment in which they occur (like location and temporal evolution) can only be resolved with complementary observations of the produced electromagnetic solar emission by several instruments (Anastasiadis 2002). The identical SREM units offer a unique opportunity to study the evolution of these processes from different geometrical perspectives in space.

However, the capabilities of SREM units to detect solar origin events have not been evaluated so far. Hence, before proceeding to any detailed timing studies and trying to understand the relevant physical processes, it is essential to first establish what kind of solar origin events SREM units can actually identify. The scope of this work is to link registered SPEs by SREM units with their originating solar energetic processes, like flares and coronal mass ejections (CMEs), by using different/complementary information from several space-borne solar observations. Such an association would be an important indication of the potential of SREM units as an integrated alarm system for space weather hazards.

Solar particle events are associated with flaring activity in complex active regions on the surface of the Sun and/or with CMEs and interplanetary (IP) shocks driven by CMEs. Solar flares are sudden, violent and very energetic explosions occurring in active regions around sunspots, which are powered 
by sudden large changes of the local magnetic field topology through reconnection processes. These processes lead to huge releases of magnetic energy (e.g. Bothmer \& Zhukov 2007), which in turn leads to plasma heating, particle acceleration and mass transport and produces electromagnetic radiation across the electromagnetic spectrum at all wavelengths from long-wave radio to the shortest wavelength gamma rays. Solar particle events associated directly with flares mostly originate from impulsive events at the western hemisphere, arrive on Earth as rapidly as some electromagnetic signatures (within tens of minutes to a couple of hours) and usually last for hours. Coronal mass ejections are ejections of material from the solar corona, primarily of protons and electrons, which are usually observed with coronagraphs like the space-borne $\mathrm{SOHO} / \mathrm{LASCO}$ instrument. Most CMEs originate from active regions and are associated with flares, range in speed from a few tens of $\mathrm{km} \mathrm{s}^{-1}$ up to $\sim 3000 \mathrm{~km} \mathrm{~s}^{-1}$ and typically reach Earth one to five days after the eruption from the Sun. There are also CMEs that are associated with filament eruptions, some caused by flaring activity in the neighboring region. CMEs interact during their propagation with the solar wind and the Interplanetary Magnetic Field (IMF). Fast CMEs eventually drive IP shocks when their speed is faster than the local magnetosonic speed. Solar particle events that are mostly related to CMEs and IP shocks originate from these fast CMEs (which may or may not be linked to solar flares), take from half a day to a couple of days to arrive on Earth and usually last for several days.

There have already been detailed studies in literature concerning characteristics and physical processes linked to SPEs (Cliver et al. 1982; Kahler 1994; Mewaldt et al. 2005; Tylka et al. 2005, and more recently from Reames 2009 \& Li et al. 2009). Similar studies concerning specific SPEs detected by SREM units will be the subject of forthcoming papers, as this is an ongoing research.

In this paper, we study SPEs registered by the SREM unit onboard INTEGRAL during four periods of solar cycle 23 with intense solar activity (October-November 2003, January and September 2005 and December 2006) and investigate their solar origin association.

\section{The Standard Radiation Environment Monitor (SREM) unit}

The SREM units have been developed in a partnership of the European Space Agency (ESA), the Paul Scherrer Institute (PSI) and Contraves Space A.G. They provide valuable data for both the near-Earth particle radiation environment (e.g. trapped particles in the radiation belts) and the interplanetary (IP) particle radiation environment with the diverse orbits of missions equipped with jointly-calibrated SREM units, offering unique opportunities for a comprehensive investigation of SPEs. Some studies based on SREM data have already been presented in literature (Hajdas et al. 2003; Mohammadzadeh et al. 2003; Evans et al. 2008).

The SREM unit consists of three silicon diode detectors (D1, D2 and D3) in a two-detector-head configuration. One system is a single silicon diode detector (D3) and the other system uses two silicon diodes (detectors D1/D2) in a co-axial configuration (detector telescope configuration). The window of the D3 detector is covered with $0.7 \mathrm{~mm}$ aluminum layer, which defines the lower energy threshold for electrons to $E>0.5 \mathrm{MeV}$ and for protons to $E>10 \mathrm{MeV}$. The D1/D2 detector is covered with a $2 \mathrm{~mm}$ aluminum layer, giving a proton and electron threshold
Table 1. List of the SREM channels and the corresponding energy ranges of detected protons and electrons in $\mathrm{MeV}$ units.

\begin{tabular}{ccccc}
\hline \hline \multirow{2}{*}{ SREM } & \multicolumn{2}{c}{ Proton energy } & \multicolumn{2}{c}{ Electron energy } \\
\cline { 2 - 5 } Channels & $E_{\min }$ & $E_{\max }$ & $E_{\min }$ & $E_{\max }$ \\
\hline TC1 & 27 & $\infty$ & 2.00 & $\infty$ \\
S12 & 26 & $\infty$ & 2.08 & $\infty$ \\
S13 & 27 & $\infty$ & 2.23 & $\infty$ \\
S14 & 24 & 542 & 3.20 & $\infty$ \\
S15 & 23 & 434 & 8.18 & $\infty$ \\
TC2 & 49 & $\infty$ & 2.80 & $\infty$ \\
S25 & 48 & 270 & - & - \\
C1 & 43 & 86 & - & - \\
C2 & 52 & 278 & - & - \\
C3 & 76 & 450 & - & - \\
C4 & 164 & $\infty$ & 8.10 & $\infty$ \\
TC3 & 12 & $\infty$ & 0.80 & $\infty$ \\
S32 & 12 & $\infty$ & 0.75 & $\infty$ \\
S33 & 12 & $\infty$ & 1.05 & $\infty$ \\
S34 & 12 & $\infty$ & 2.08 & $\infty$ \\
\hline
\end{tabular}

of 20 and $1.5 \mathrm{MeV}$, respectively. However, the shielding between the two diodes in the telescope allows the passage only for protons with energies greater than $43 \mathrm{MeV}$, depositing energy in both detectors. Thus, using the two diodes in coincidence gives pure proton count-rates. The energy of detected particles is binned into 15 channels, each with its own proton and electron energy response, which are presented in Table 1. Since SREM samples the spectra in broad and overlapping energy bands (e.g. channels TC1, S12 and S12 or TC3, S32, S33 and S34), the count-rates of the channels are strongly dependent on each other, e.g. counts in channel S15 are also counted in channels S14, S13, S12 and TC1. As Table 1 clearly indicates, all channels are sensitive to protons, there are no pure electron channels, while coincidence channels $\mathrm{C} 1-\mathrm{C} 4$, and channels S25 and the S15 can be considered as pure proton channels. However, in the presence of high energetic electrons with energies above $8 \mathrm{MeV}$, channel C4 can be contaminated by electron counts. We refer the reader to the papers by Evans et al. (2008), Sandberg et al. (2009) and Bühler et al. (1996) for further details concerning the characteristics of SREM units. Information about the associated spacecraft and instrument geometries, calibration and response functions of the SREM channels can be retrieved from the Paul Scherrer Institute SREM site (http: //srem . web . psi . ch).

\section{Observations}

Below we give an overview of the selected SREM and solar data necessary for the present study of SPEs.

\subsection{Selected SPEs recorded by SREM units}

We considered only SPEs that occurred during four time intervals of cycle 23 characterized by intense solar activity: October-November 2003, January 2005, September 2005 and December 2006. Only data recorded by the SREM unit on board INTEGRAL are considered since the satellite was closer to Earth, with a better defined orbit than ROSETTA and no data gaps were present like in the PROBA/SREM recordings. 
K. Tziotziou et al.: Using a new set of space-borne particle monitors to investigate solar-terrestrial relations
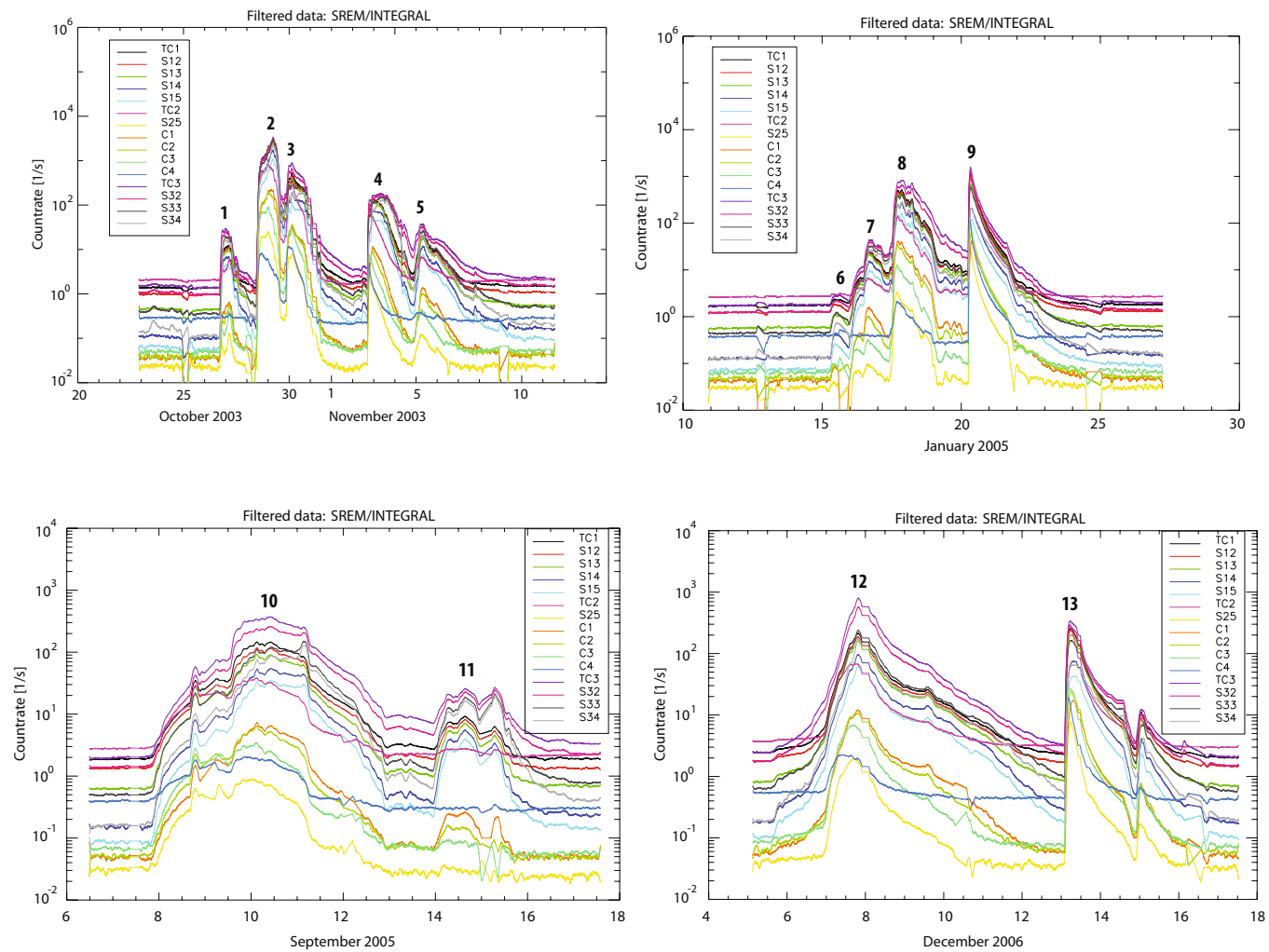

Fig. 1. Particle counts recorded in different channels of the SREM unit onboard INTEGRAL for the four selected periods of time. Numbers indicate the thirteen identified SPEs shown in Table 2, while the energy intervals of SREM channels are given in Table 1.

Figure 1 shows the particle counts as they were recorded in different channels of the SREM unit onboard INTEGRAL for the four selected time intervals. The presented data were filtered to remove count rates attributed to radiation belt passages. The recorded particle counts show several peaks that correspond to SPEs. Using Fig. 1, we can resolve some of their characteristics such as a) the onset of the event; b) the time of the local maxima; and c) an estimated value of the maximum flux for selected channels. The onset is determined as the time when the countrates of a selected channel start to increase exponentially. For the calculation of the maximum flux the simple conversion factor method (Evans et al. 2008) can be used, which gives a good estimate for the fluxes; more advanced methods are discussed by Sandberg et al. (2009). Table 2 summarizes the characteristics of the registered thirteen SPEs which are numbered in Fig. 1. Both onset times and maximum times were derived using $\mathrm{C} 1$ channel measurements, which correspond to pure $43-86 \mathrm{MeV}$ protons. However, as we have already mentioned in Sect. 2, the count-rates of the channels are strongly dependent on each other. Therefore the recorded countrates in most channels have a qualitatively similar behavior concerning both onset and maxima occurrence. We also note that some of the recorded SPEs are very complex (i.e. SPE 10 in September 2005), comprising in reality several events, and they will be further discussed in this paper.

\subsection{Solar data}

For the analysis presented in this paper we used several space instruments that provide radio/optical/X-ray (ROX) observations of solar flares and CMEs, which are considered to be the solar/IP origin of SPEs. In particular we have identified, retrieved and reduced observations from the following space instruments:

- The X-ray Sensor (XRS) oboard GOES ${ }^{1}$ satellites which measures whole-sun X-ray fluxes in two wavelength bands (0.5-4 $\AA$ and 1-8 $\AA$ ) in real time (see Fig. 3, top panel for a recorded example). The GOES/XRS measurements are ideal to detect the beginning and evolution of solar flares and has been used by the solar community as the main alert instrument for solar flares.

- The Transition Region and Coronal Explorer (TRACE), which provides continuous two-dimensional coverage of phenomena related to the solar photosphere, chromosphere, transition region and corona with high spatial and temporal resolution in several ultraviolet (UV) and extreme ultraviolet (EUV) wavelengths (Handy et al. 1999). TRACE has a "flare watch" mode which offers two-dimensional time-series images with high temporal cadence when a flare occurs. These images can be used to a) study in detail both the spatial and temporal evolution of the flare, providing valuable information about the onset and peak of the event on the solar surface and b) to obtain the intensity variation during the event, which relates to some of the characteristics of the recorded by SREM SPE flux profile (see Fig. 2 for recorded examples).

- The Large Angle and Spectrometric Coronagraph (LASCO), the Charge Element and Isotope Analysis System/Solar Extreme-Ultra-Violet Monitor (CELIAS/SEM) and the

\section{${ }^{1}$ See}

http://rsd.gsfc.nasa.gov/goes/text/goes. databook.html for information concerning the GOES satellites. 
A\&A 514, A21 (2010)

Table 2. The proton flux recorded by channel $\mathrm{C} 1$ of the SREM unit onboard INTEGRAL satellite for the maximum of the events.

\begin{tabular}{cccccc}
\hline \hline \multirow{2}{*}{$\begin{array}{c}\text { Event } \\
\text { number }\end{array}$} & \multicolumn{2}{c}{ Onset } & \multicolumn{2}{c}{ Maximum } & \multirow{2}{*}{\begin{tabular}{c} 
Flux $43-86 \mathrm{MeV}$ \\
nyynnn \\
\cline { 2 - 4 } counts/cm $/ \mathrm{s})$
\end{tabular}} \\
\hline 1 & $26 / 10 / 2003$ & $19: 16: 46$ & $27 / 10 / 2003$ & $04: 24: 54$ & 104.23 \\
2 & $28 / 10 / 2003$ & $11: 36: 47$ & $29 / 10 / 2003$ & $03: 40: 47$ & 27055 \\
3 & $29 / 10 / 2003$ & $18: 22: 37$ & $30 / 10 / 2003$ & $02: 47: 40$ & 5132.5 \\
4 & $02 / 11 / 2003$ & $17: 45: 29$ & $02 / 11 / 2003$ & $22: 23: 04$ & 1769.29 \\
5 & $05 / 11 / 2003$ & $04: 08: 46$ & $05 / 11 / 2003$ & $05: 38: 58$ & 189.55 \\
6 & $15 / 01 / 2005$ & $06: 40: 12$ & $15 / 01 / 2005$ & $16: 46: 27$ & 14.60 \\
7 & $16 / 01 / 2005$ & $05: 26: 02$ & $16 / 01 / 2005$ & $16: 27: 24$ & 220.14 \\
8 & $17 / 01 / 2005$ & $13: 52: 04$ & $17 / 01 / 2005$ & $20: 09: 51$ & 5263.32 \\
9 & $20 / 01 / 2005$ & $07: 42: 16$ & $20 / 01 / 2005$ & $08: 55: 25$ & 5937.36 \\
10 & $07 / 09 / 2005$ & $14: 11: 01$ & $10 / 09 / 2005$ & $02: 37: 32$ & 951.42 \\
11 & $13 / 09 / 2005$ & $17: 43: 23$ & $14 / 09 / 2005$ & $07: 52: 09$ & 48.30 \\
12 & $05 / 12 / 2006$ & $13: 06: 23$ & $07 / 12 / 2006$ & $17: 41: 55$ & 1452.44 \\
13 & $12 / 12 / 2006$ & $20: 10: 55$ & $13 / 12 / 2006$ & $08: 05: 24$ & 2018.04 \\
\hline
\end{tabular}

Notes. Event numbers correspond to respective SPEs marked in Fig. 1.
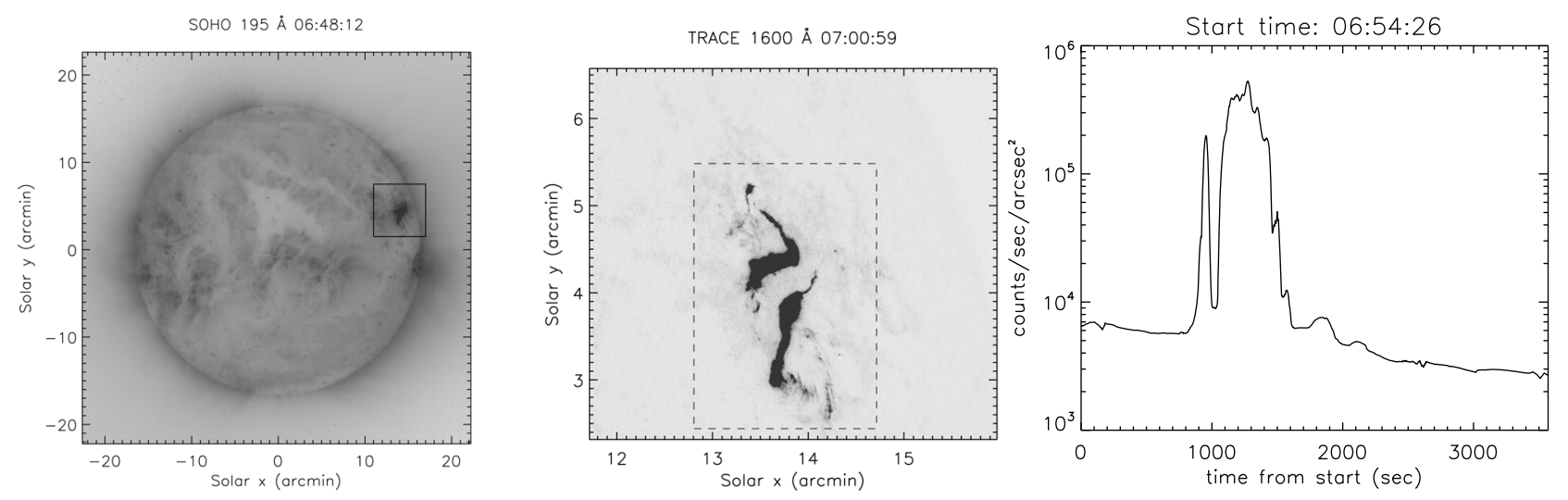

Fig. 2. Plots corresponding to the January 20, 2005 X-class flare. Both SOHO/EIT and TRACE images are in reversed color table for clarity (black corresponds to higher intensities). Left panel: full Sun $195 \AA$ AOHO/EIT image (the black box shows the location of the flare). Middle panel: $1600 \AA$ TRACE image corresponding to the box area shown in the left SOHO/EIT image. The dashed rectangular box shows the integration region for the flux curve shown in the right panel. Right panel: the calculated intensity flux originating within the dashed rectangular box, shown in the TRACE image in the middle panel.

Extreme ultraviolet Imaging Telescope (EIT) onboard the Solar and Heliospheric Observatory $(\mathrm{SOHO}$ ) (Domingo et al. 1994, 1995). SOHO has been one of the most successful solar missions, studying the structure and dynamics of the Sun with a payload that consists of 12 different instruments, including the aforementioned three instruments that were used in our analysis. LASCO (Brueckner et al. 1995), which is a set of three coronagraph telescopes used for the observation of the solar corona from 1.1 to 32 solar radii, is used in the present analysis for the detection (onset and evolution) of CMEs attributed to solar flares and sometimes to filament eruptions. CELIAS/SEM (Hovestadt et al. 1995) is a spectrometer designed to continuously measure the full solar disk absolute solar flux at the prominent He II $30.4 \mathrm{~nm}$ line as well as the absolute integral flux between 17 and $70 \mathrm{~nm}$, which coincides with its Al filter band-pass. The almost continuous in time CELIAS/SEM data can be used to identify the onset and evolution of SPEs as well as the presence of IP shocks and have been proven very useful for inter-calibration of the EIT instrument (see Fig. 3, second panel for a recorded example). Finally EIT (Delaboudinière et al. 1995) obtains full-disk images of the solar transition region and inner corona in four EUV bandpasses, offering useful information mostly about the location, but also for the temporal evolution - albeit in low resolution - of flares (see Fig. 2 for a recorded example).

- The Reuven Ramaty High Energy Solar Spectroscopic Imager (RHESSI), which provides simultaneous, high resolution imaging and spectroscopy of solar flares from soft $\mathrm{X}$-rays to gamma rays with high time resolution (see Fig. 3, third panel for a recorded example), used for the investigation of particle acceleration and energy release in flares (Lin et al. 2002).

- The WAVES instrument onboard WIND satellite (Bougeret et al. 1995), which provides measurements of the radio and plasma wave phenomena which occur in geospace, in the frequency range from a fraction of one Hertz up to about $14 \mathrm{MHz}$ for the electric field and $3 \mathrm{kHz}$ for the magnetic field. The instrument has five main receiver systems, but for this study we only used the Thermal Noise Receiver 
(TNR), which covers the frequency range $4 \mathrm{kHz}$ to $256 \mathrm{kHz}$, the Receiver Band 1 (RAD1) with a frequency range of $20 \mathrm{kHz}$ to $1040 \mathrm{kHz}$ and a bandwidth of $3 \mathrm{kHz}$ and the Radio Receiver Band 2 (RAD2), which is similar to RAD1 but with a bandwidth of $20 \mathrm{kHz}$ and a much higher frequency range of $1.075 \mathrm{MHz}$ to $13.825 \mathrm{MHz}$. WIND/WAVES radio data are useful for the detection of pre-flare activity and the onset of the flare, which are usually associated with type III radio bursts and for the detection of accompanying CMEs and IP shocks which are related to type II radio burst signatures (see Fig. 3, bottom panel for a recorded example).

For further details on the instruments and the data they record, we refer the reader to the corresponding references.

Available ROX data during the selected four temporal periods were downloaded and reduced using standard IDL Solarsoft routines or newly written IDL routines. Availability of the aforementioned data depends on several factors like the satellite's observing target, the availability of the instrument due to technical problems or maintenance reasons (e.g. SOHO/EIT CCD bakeout periods) and to spacecraft/instrument eclipse periods (e.g. TRACE, RHESSI) or passages through the South Atlantic Anomaly (e.g. RHESSI). Downloading and reduction of data consisting of time series of two-dimensional images (e.g. $T R A C E, S O H O / E I T)$ was restricted to solar events corresponding to X-class flares due to the large amount and huge size of available data, while particular emphasis has been given to the proper reduction, calibration and co-alignment of these data.

All acquired solar observations are used in a complementary way to each other to derive the association of SPEs with a particular flare and/or any accompanying CME. This is necessary because both spatial and temporal information cannot always be derived from observations obtained with a single instrument and observations from certain instruments/satellites are not available for all time periods, as mentioned before.

Below we present some examples of recorded solar activity corresponding to the X-class 2005 January 20 flare. Figure 2 shows images of the X7.1 flare obtained with SOHO/EIT (left panel) and TRACE at $1600 \AA$ (middle panel) as well as the calculated $1600 \AA$ intensity flux profile (right panel). Figure 3 shows the corresponding GOES X-ray fluxes (top panel), CELIAS/SEM He II $30.4 \mathrm{~nm}$ flux (second panel), RHESSI lightcurves (third panel) and WIND/WAVES radio spectra (bottom panel). These observations were used as follows to derive the SPE association discussed in Sect. 4.2:

- The GOES/XRS observations are the first indication for the occurrence of a flare and its evolution. The recorded X-ray flux (Fig. 3, top panel) shows an important X-class flare (X7.1) peaking around 07:00 UT, but it does not provide any information about the actual location of the flare.

- The location of the flare on the solar disk can be obtained from the full Sun SOHO/EIT image (Fig. 2, left panel) and/or the TRACE observations (Fig. 2, middle panel). As these figures show, this flare occurred close to the western solar limb slightly north from the equator (N14W61).

- Temporal information about the onset and dynamics of the flare (e.g. peak time, decay etc) can be obtained from a) GOES/XRT; b) TRACE; c) CELIAS/SEM; d) RHESSI; and e) WIND/WAVES observations. Unfortunately SOHO/EIT is not suitable for any useful temporal information due to its low temporal cadence. TRACE usually offers unique highcadence image sequences ( $2 \mathrm{~s}$ cadence for this particular flare), obtained during its "flare watch mode" (see Fig. 2, middle panel). These series of two dimensional images allow
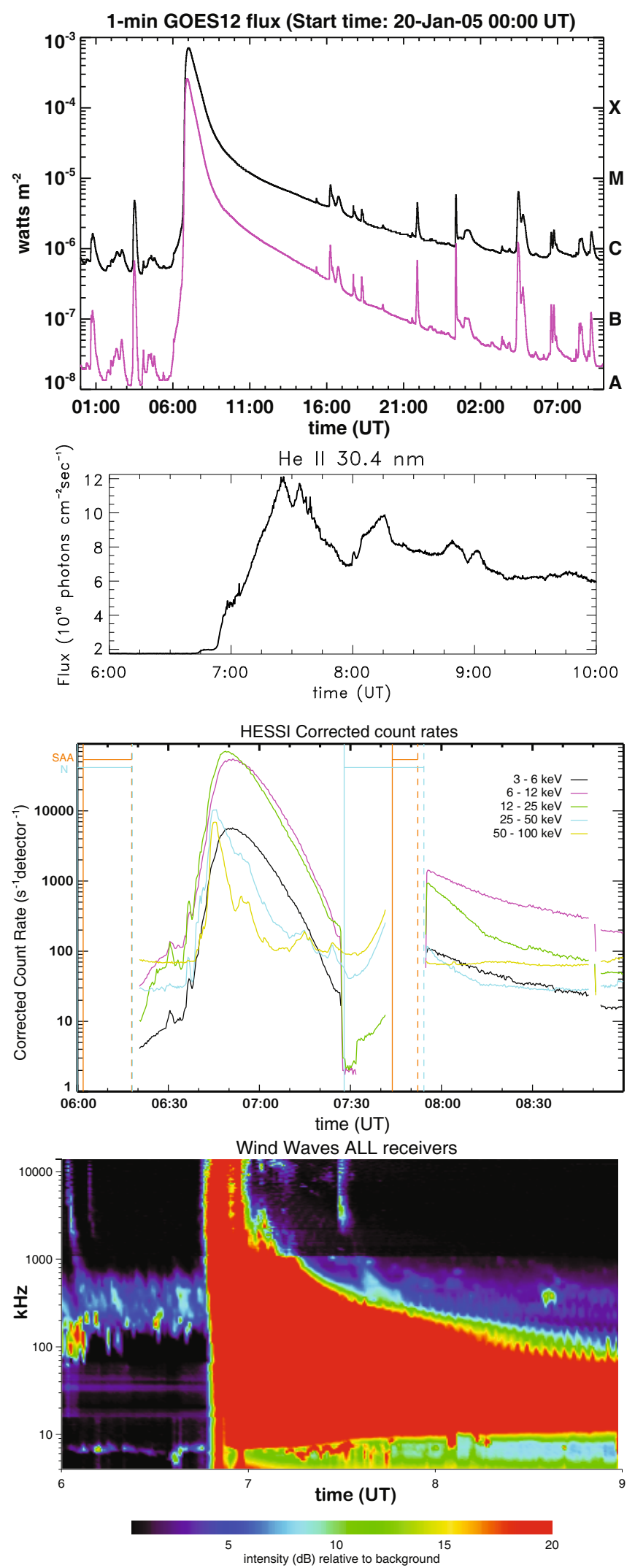

Fig. 3. Top panel: the recorded GOES X-ray fluxes at 1-8 $\AA$ (top black line) and 0.5-4 $\AA$ (bottom magenta line) for the 2005 January 20 X7.1 flare. Second panel: the corresponding CELIAS/SEM He II $30.4 \mathrm{~nm}$ flux. Third panel: the recorded RHESSI lightcurves for the same flare. Gaps in the data correspond to night (eclipse) and South Atlantic Anomaly passages. Bottom panel: corresponding radio spectra from the WIND/WAVES instrument. 
the study of the morphology (two ribbon flare in this case) and spatial evolution of the flare, which are irrelevant for the present study, but extremely important for any future detailed study of any event. Temporal variations, including onset and peak times, can be determined from the constructed $1600 \AA$ intensity variation, shown in Fig. 2 (right panel), which represents the integrated counts per unit area and time over the flare region (rectangular box of Fig. 2, middle panel) as a function of time.

Onset and peak times in different energy bins can be also determined from the RHESSI lightcurves (see Fig. 3, third panel) and the He II $30.4 \mathrm{~nm}$ flux measured with CELIAS/SEM (Fig. 3, second panel).

Finally, in WIND/WAVES radio spectra (Fig. 3, bottom panel) the onset of the flare is revealed by the presence of type III radio bursts, which accompany the impulsive phase of large flares and show as radio bursts rapidly drifting from high to low frequencies. Type III bursts are signatures of small-scale energy release in the form of energetic electrons beams that escape along open field lines.

It is clear from the presented figures that onset and peak times can be rather different because different data reflect different physical processes and properties of the source. However, we should stress once again that the scope of the present paper is not a precise timing study, but the association of recorded SPEs with their solar origin sources.

- A companion to the flare CME can be revealed directly by $L A S C O$ images (not shown here), indirectly by WIND/WAVES radio spectra and sometimes by CELIAS/SEM observations. The two dimensional sequence images registered by the $L A S C O$ coronographs can be used to verify the presence of a CME, determine the morphology of the CME and to calculate its onset time and linear speed. For this particular flare, available $L A S C O$ images showed that there was a halo CME released with a linear speed of $882 \mathrm{~km} \mathrm{~s}^{-1}$.

In WIND/WAVES radio spectra (Fig. 3, bottom panel), type II radio bursts are strongly associated with fast CMEs and interplanetary shock waves. They show up as a radio emission drift towards lower frequencies due to decrease of the plasma density as the shock propagates away from the Sun and hence can provide information about both the outward propagation speed and the origin of shocks.

Finally the presence of CMEs can be sometimes revealed in CELIAS/SEM observations (see Fig. 3, second panel) as a visible enhancement in the He II flux well after the onset of the flare.

The aforementioned scheme was used for the study of all solar data associated to the SPE observations. It is obvious that the reduced solar data contain far more information than for the scope of this paper. A detailed study of specific events is essential to understand the physics involved, but it is an ongoing research, to be presented in future works.

\section{Association of SPEs with solar events}

For the precise association of SPEs with solar events there are a number of facts that have to be considered:

1. During the explosive energy release of solar flares, particles and especially protons are accelerated to very high velocities. These particles do not travel towards Earth on a straight line, but along the Sun's extended magnetic field lines, the interplanetary magnetic field (IMF), which has a structure resembling a spiral.
2. Due to the aforementioned spiral topology of the IMF, the flare location on the surface of the Sun affects both the intensity and the arrival time of these high speed particles on Earth. Particle fluxes originating from flares that occur in the center of the Sun's disk, have a completely different behavior than particle fluxes from flares occurring at the solar limb. Solar particle events originating from the West limb are usually impulsive-like events, showing a fast intensity rise directly reflecting the flare profile, while SPEs originating from the East limb and the center of the solar disk usually show a more gradual intensity increase (Reames 1999). Both the arrival itself of particles on Earth and their arrival time depend ultimately on the magnetic connectivity of the flare site to Earth and also largely on the particle speed.

3. The arrival time for most energetic particles can vary from a couple of hours to almost a day (or sometimes even days), depending both on the location of the flare and the energy (velocity) of the released and accelerated particles.

Taking into account the aforementioned facts and the available information collected from the analysis of all available ROX observations we established associations for all recorded SPEs with solar events. The correlations were mostly done using the time of the maximum SREM flux, because the onset time is quite subjective, as it depends on how it is defined. Furthermore, most of the time it is also affected not only by the major solar event that caused the particular maximum peak, but also by preceding SPEs. In the latter case, when another major SPE occurred within the previous couple of days, the flux does not manage to reach background level before the new event occurs. Sometimes, even lower class flare events ( $\mathrm{M}$ and $\mathrm{C}$ class flares) occur, which can also cause an increase in the particle flux before a major SPE starts. The established associations are collectively shown in Table A.1, which also indicates (in bold letters) the major solar event of each SPE. Below we discuss in further detail the obtained associations. Start and peak times of flares correspond to the respective times of $G O E S / X R S$ observations.

\subsection{The October-November 2003 SPES}

This period is characterized by five intense SPEs shown in Fig. 1 and Table 2 (events 1 to 5).

The first event seems to mainly correlate with the X1.2 flare that occurred on October 26, 2003 in AR10484 and is also associated with a fast CME. The flaring region was close to the solar equator and to the western part of the solar disk, but closer to disk center than the limb (N02W38), which explains the ten hour delay in the recorded particle flux and also the rather low flux of particles. The rather broad SPE peak though, as well as the almost 1.5 day decline of the SPE flux after the recorded maximum contains also contributions from a series of four $\mathrm{M}$ class flares from the same region that occurred within $14 \mathrm{~h}$ after the $\mathrm{X}$ class flare, three of them also associated with CMEs.

The second event correlates with the massive 2003 October 28 X17 flare that occurred in AR10486 accompanied by a very fast halo CME. Although the flaring region was very close to the solar disk center and slightly on the eastern part of it (S16E08), it was such an energetic event that the SPE flux onset is less than half an hour later, while it took only ten hours for the SPE flux to peak, exhibiting the highest recorded flux by INTEGRAL/SREM during all four time periods of the study. The recorded SPE flux decreased quite rapidly in half a day, because there was no other important solar event afterwards associated with a CME (only two M class flares in the next $18 \mathrm{~h}$ with 
no recorded CME), but most importantly because the third SPE event started before the particle flux decreased to background level.

The 2003 October 29 X10 flare in AR10486, which was accompanied by a fast halo CME, is associated with the third SPE event. The flare occurred almost at disk center (S15W02) and gave an SPE flux that peaked six hours later. The rather high recorded flux is partly an accumulation result since the flux of the previous event did not have time to recover to background level and was still rather high when the onset of the third event occurred. The recorded decline to almost background level is rather long (three days), showing that very energetic events can affect Earth for several days. There were hardly any other important CME related flare events during the decline period.

The fourth event mainly correlates with the 2003 November 2 X8.3 flare that occurred again in AR10486 and a very fast halo $\mathrm{CME}$. The flaring region was then located on the western part of the solar disk (S14W56). The recorded SPE flux started increasing from background level and peaked just five hours later, reaching a high flux level in intensity. The rather broad peak and the decline phase, which lasted almost two days, contained contributions from two other lower intensity X-class flares, which occurred on November 3, 2003 in AR10488, slightly to the north of AR10486. These flares occurred within less than $16 \mathrm{~h}$ after the main flare and were both associated with CMEs. Again the rather slow recovery did not reach background level before the onset of the fifth SPE of this period.

The final SPE of this period corresponds to the largest X-class flare event of this period, the 2003 November 4 X28 flare in AR10486. This mega-flare, which was accompanied by a very fast halo CME, occurred right on the west limb of the Sun (S19W83), which explains the main characteristics of the recorded SPE flux. The increase of the flux was rather smooth and not impulsive-like, and the particle flux peaked ten hours after the flare. The maximum recorded counts are rather low for such an energetic event, which is to be expected though because the event originated from the west limb, which has in principle the worst magnetic connectivity with Earth. The decrease to background level was rather long, lasting almost four days. We point out that this is a rather clean event, in the sense that no other important flares occurred after the X-flare (only two M-class events with no recorded CMEs), and hence all the recorded SPE flux can be attributed to particles blown away by the X28 flare.

\subsection{The January 2005 SPES}

In January 2005 the INTEGRAL/SREM unit recorded mainly three strong SPEs (7 to 9 in Table 2) and a fourth rather week event (event No 6).

It is not that clear which solar flare the first SPE (event No 6) is associated with. In any case, the event is attributed to AR10720, which was located close to solar disk center (N14E08). This region gave the 2005 January 15 X1.2 flare, but with no CME recorded by SOHO/LASCO. The recorded SPE flux most probably correlates with this flare, but there have been five more M-class flares that occurred in the same region in the preceding $22 \mathrm{~h}$, one of which (the 2003 January 14 M 1.0 flare at 16:06 UT) was also associated with a CME. This event is anyway very weak compared to the other events of this period, with a very fast decline to almost background level in something more than half a day.

The second event (No 7) mostly correlates with the 2005 January 15 X2.6 flare that occurred in AR10720 accompanied by a very fast halo $\mathrm{CME}$. The flaring region was very close to the solar disk center (N14W08), and it took almost $17 \mathrm{~h}$ for the SPE flux to peak. The increase of the SPE flux, which started around eight hours after the flare, is rather smooth, followed by an impulsive-like increase eight hours later. This smooth increase is probably due to a series of M-class flares that occurred within $19 \mathrm{~h}$ preceding the X-flare, all but one from the same region. Two of these M-class flares were accompanied by CMEs, the 2005 January 15 M 8.6 flare, which had a very fast recorded halo CME and the M3.2 flare, which occurred in AR10718 and also produced a CME. The first one is probably responsible for a small peak recorded by some of the SREM channels hours before the large SPE. The recorded SPE flux decreased within something more than a day, but not to background level, since the next SPE took place before complete recovery.

The 2005 January 17 X3.8 flare in AR10720 is associated with the eighth SPE. This flare occurred close to disk center on the western part of the solar disk (N15W25) and is rather complex. It was related to two very fast halo CMEs that happened within $25 \mathrm{~min}$, the second of which was much faster than the first and probably caught up with it in interplanetary space, where they produced IP shocks. The SPE flux peaked $10.5 \mathrm{~h}$ after the X-flare, while the decline phase lasted almost two and a half days, with a slow decrease the first day followed by a faster decline over half a day and then again a much slower one-daydecline, before the next SPE occurred. The third much slower decline has to do with three flares, an M 6.7, an X1.3 flare and an M 2.7 flare all in AR10720, which all occurred on January 19, 2005 within three hours and were all associated with four CMEs in total, including one very fast halo CME.

The final event of this period (SPE event No 9) corresponds to the largest 2005 January 20 X7.1 flare, which occurred in AR 10720. It took place on the western part of the solar disk (N14W61) and was accompanied by a rather slow CME. However, the onset of the SPE flux was rather quick and it also peaked very quickly within two hours after the flare, indicating a very good magnetic connectivity of the event location with Earth. The decrease to background level lasted almost three days and was very smooth and clean, because only one M-class flare accompanied by a slow CME occurred during the decline phase.

\subsection{The September 2005 SPES}

During this period the INTEGRAL/SREM unit recorded two rather complex SPEs, with the first one (event No 10) being extremely broad, as can be seen in Fig. 1. They are all related to just one active region, AR10808, but several different flares.

The real onset of SPE event No 10 seems to be on September 8, 2005, peaking on September 10 and lasting until late evening September 13 when the next SPE took place before the flux reached background level again. This is a very complex SPE that corresponds to several X-class and M-class flares that occurred during this time interval, which unfortunately are very difficult to disentangle. However, signatures of all X-class flares are apparent in the recorded flux. We will only qualitatively discuss these X-class flare events because it is impossible to discern the contribution of the many M-class flare events on the recorded SPE flux, a task which is further complicated because there are no SOHO/LASCO CME data available until late September 9, 2005. Anyway, as the majority of the flares occurred on the East limb, one expects a rather short delay between the flare and the peaks of the SPE flux if any CMEs were released. The first rather smooth increase of the SPE flux for the first $18-20 \mathrm{~h}$ of September 8, 2005 is probably related to the 
2005 September 7 X17 flare that occurred in AR10808, right on the east limb, as well as probably to other flares that this region produced on the back side of the Sun before appearing on the limb. Then late in September 8, 2005 there was a small abrupt increase in the recorded flux that was probably associated with the X5.4 flare, which occurred on the Sun a couple of hours before. The first $12 \mathrm{~h}$ of September 9, 2005 there was another flux increase, which was probably associated with the X1.1 and X3.6 flares that occurred during that period. The much higher increase of SPE flux recorded by the afternoon/evening of September 9, 2005 was probably mainly associated with the X6.2 flare, which occurred that evening and also had a very fast halo CME (the first one recorded after a $S O H O / L A S C O$ two-day lack of recordings). The second 2005 September 10 X2.1 flare, which took place around 22:00 UT and also had a fast halo CME associated with it, probably appeared as a peak increase in the SPE flux in a couple of channels around midnight when the new day started. Afterwards there was a gradual decline for almost three days, until late evening of September 13, 2005 because there were no further X-class flare events, but several M-class flares with only a couple of them with recorded CMEs.

The eleventh SPE event, which seems to have an onset in the evening of September 13, 2005 appears to be associated with an X1.5 flare and an X1.7 flare that occurred four hours later that evening, both associated with CMEs. The first CME was rather fast, while the second was much slower, something that probably explains the rather extended peak in the flux, which covered almost the whole day of September 14, 2005. Finally the second clear peak seen in the SPE flux just before midday on September 15, 2005 was associated with the X1.1 flare that occurred on the same day, which however had no registered CME (there was only one CME associated with an M 1.3 flare that occurred six hours before). The decline of the flux to background level was very fast afterwards, lasting merely one day, since there were no other important CME-related events happening during that period.

\subsection{The December 2006 SPEs}

During this temporal period the INTEGRAL/SREM unit recorded two strong SPEs (events No 12 and 13), which are shown in Fig. 1 and are both related to one single region, AR10930. The first SPE event of this period is quite extended.

The twelfth SPE shows an increase of flux in most channels, starting the midday of December 5, 2006. This increase is probably associated with the X9.0 flare of December 5, 2006, however with no registered CME, since $\mathrm{SOHO} / \mathrm{LASCO}$ was not operating during this time. Since there is no large increase in flux, one probably has to assume that no important CME was produced during this flare eruption. The major flux increase occurred at midnight on December 7 to 8, 2006 and is associated with the X6.5 flare that occurred on December 6, 2006. This flare occurred in the eastern part of the solar disk (S06E63) close to the equator. Again no SOHO/LASCO data were available and the SPE flux peaked almost $22 \mathrm{~h}$ later. This flare was followed by two M-class flares within the next $24 \mathrm{~h}$, which were accompanied by slow CMEs. However, the very long flux decline, which was very smooth and lasted almost five days, together with the fact that no other major events were present during this period indicates that the $\mathrm{X}$ flare and probably the two $\mathrm{M}$ flares that followed produced great numbers of energetic particles.

The final SPE (event No 13) is a rather clean event associated mainly with two flares. It starts with a sudden increase in flux, almost to the same level as the previous event, which is associated with the X3.4 flare that occurred on December 13, 2006. AR10930 was then located in the western part of the solar disk (S06W23) and also produced a fast halo CME. The SPE flux peaked six hours later and started decreasing smoothly and slowly for a couple of days, when no other, not even M-class flare events, occurred. Then another X1.5 flare occurred on December 14, 2006 with a rather slow halo CME that appeared as a clear peak in the recorded SPE flux at midnight on December 15, 2006. Afterwards the SPE flux gradually declined to background level relatively fast, within one and a half days.

\section{Conclusions}

The goal of the present study was to establish a first link of individual SREM recorded SPEs with their potential solar sources to evaluate the capabilities of SREM for such investigations. As our analysis indicated, SREM is a reliable particle radiation monitor able to detect major solar particle events associated with large $\mathrm{X}$-class and lower M-class flares and possible accompanying CMEs. It is remarkable that even for complex events, like that of the September 2005 period, which is a combination of several $\mathrm{X}$ - and M-class flare events, the recorded SREM data clearly show identifiable signatures of all X-class flare events.

More precisely, our extensive analysis of several ROX solar data indicated clear associations with X-class solar flares and CMEs for the SPE events that occurred during the OctoberNovember 2003, January 2005 and December 2006 periods, which were marked by intense solar activity. For the September 2005 period, associations with X-class flares and CMEs were possible but not straightforward due to the complexity of the SREM-recorded SPEs, as we already explained.

Follow-up work will include a detailed temporal and spatial study of ROX data associated with specific SPEs to understand the complex physics involved in the generation, dynamics and propagation of solar energetic particles in IP space. Furthermore, future research should also focus on the inter-comparison of SPEs recorded by SREM units with relevant solar data and time sequences to investigate how the physical and temporal characteristics of the SEP sources affect the recorded SEP profiles. The presence of jointly-calibrated SREM units in several missions with diverse orbits and especially on IP missions like ROSETTA, offers a unique opportunity for a comprehensive spatial and temporal investigation of SPEs. The spatial distribution of SREM units in geospace and IP space can prove extremely useful, especially for complex events like the September 2005 events, since they operate on different satellites. These events need to be further investigated. For example the corresponding ROSETTA/SREM recordings for the September 2005 SPEs look quite different due to the location of the satellite in IP space at the time when the events occurred.

Because all major solar flare events during the investigated time intervals had corresponding signatures at SREM units, we conclude that SREM units can prove to be a valuable tool for monitoring and studying the near-Earth and the IP particle radiation environments. SREM measurements have the potential to become a valuable asset of an integrated alert system to be used for space weather nowcasts and forecasts, critically needed for the mitigation of space weather hazards (Daglis 2004).

Acknowledgements. This work has been supported through the ESA Contract $21480 / 08 / \mathrm{NL} / \mathrm{NR}$. We would like to thank the anonymous referee for comments and suggestions that helped to improve the paper. 
K. Tziotziou et al.: Using a new set of space-borne particle monitors to investigate solar-terrestrial relations

\section{Appendix A: Association of SPEs with solar events}

Table A.1. Association of SPEs with solar events (flares and CMEs). ${ }^{1}$

\begin{tabular}{|c|c|c|c|c|c|c|c|c|c|}
\hline \multirow[b]{2}{*}{ Event } & \multicolumn{6}{|c|}{ Flare event } & \multicolumn{3}{|c|}{ Coronal mass ejections } \\
\hline & Date & $\begin{array}{l}\text { Start time } \\
\text { (UT) }\end{array}$ & $\begin{array}{l}\text { Peak time } \\
\text { (UT) }\end{array}$ & Flare class & Location & AR No & $\begin{array}{c}\text { Observed time } \\
\text { (UT) }\end{array}$ & $\begin{array}{c}\text { PA } \\
\text { (degrees) }\end{array}$ & $\begin{array}{c}\text { Linear speed } \\
\left(\mathrm{km} \mathrm{s}^{-1}\right)\end{array}$ \\
\hline \multirow[t]{8}{*}{1} & 2003 Oct. 26 & $17: 21$ & $18: 19$ & $\mathrm{X} 1.2$ & N02W38 & 10484 & $17: 54: 05$ & 270 & 1537 \\
\hline & 2003 Oct. 26 & $21: 34$ & $21: 40$ & M 7.6 & N01W38 & 10484 & & & \\
\hline & 2003 Oct. 27 & $04: 12$ & 04:39 & M 1.2 & N00W44 & 10484 & 04:30:05 & 303 & 481 \\
\hline & & & & & & & 05:06:06 & 130 & 323 \\
\hline & 2003 Oct. 27 & $07: 51$ & $08: 33$ & M 2.7 & N00W45 & 10484 & 08:30:05 & 265 & 1322 \\
\hline & 2003 Oct. 27 & 09:21 & 09:27 & M 5.0 & S16E26 & 10486 & & & \\
\hline & 2003 Oct. 27 & $12: 27$ & $12: 43$ & M 6.7 & & 10486 & $13: 31: 47$ & 317 & 1005 \\
\hline & 2003 Oct. 27 & $21: 46$ & $21: 51$ & M 1.9 & N08E09 & 10488 & & & \\
\hline \multirow[t]{3}{*}{2} & 2003 Oct. 28 & 09:51 & 11:10 & X17. & & 10486 & 11:30:05 & Halo & 2459 \\
\hline & 2003 Oct. 29 & $00: 26$ & $01: 51$ & M 1.1 & & 10486 & & & \\
\hline & 2003 Oct. 29 & 04:08 & 05:11 & M3.5 & S17E06 & 10486 & & & \\
\hline 3 & 2003 Oct. 29 & 20:37 & $20: 49$ & X10. & S15W02 & 10486 & 20:54:05 & Halo & 2029 \\
\hline \multirow[t]{3}{*}{4} & 2003 Nov. 2 & 17:03 & $17: 25$ & X8.3 & S14W56 & 10486 & 17:30:05 & Halo & 2598 \\
\hline & 2003 Nov. 3 & 01:09 & 01:30 & $\mathrm{X} 2.7$ & N10W83 & 10488 & 01:59:24 & 304 & 827 \\
\hline & 2003 Nov. 3 & $09: 43$ & $09: 55$ & X3.9 & N08W77 & 10488 & 10:06:05 & 293 & 1420 \\
\hline 5 & 2003 Nov. 4 & 19:29 & 19:50 & $\mathrm{X} 28$ & S19W83 & 10486 & 19:54:05 & Halo & 2657 \\
\hline \multirow[t]{7}{*}{6} & 2005 Jan. 15 & 00:22 & $00: 43$ & $\mathrm{X} 1.2$ & N14E08 & 10720 & & & \\
\hline & 2005 Jan. 15 & 04:09 & $04: 16$ & M 1.3 & N11E08 & 10720 & & & \\
\hline & 2005 Jan. 15 & $04: 26$ & $04: 31$ & M 8.4 & N14E06 & 10720 & & & \\
\hline & 2005 Jan. 15 & $05: 54$ & $06: 38$ & M 8.6 & N11E06 & 10720 & 06:30:05 & Halo & 2049 \\
\hline & 2005 Jan. 15 & $11: 41$ & $11: 48$ & M 1.2 & N14E02 & 10720 & & & \\
\hline & 2005 Jan. 15 & $14: 08$ & $14: 23$ & M 3.2 & S08W12 & 10718 & 14:54:05 & 222 & 498 \\
\hline & 2005 Jan. 15 & $22: 01$ & $22: 08$ & M 1.0 & N14W07 & 10720 & & & \\
\hline \multirow[t]{3}{*}{7} & 2005 Jan. 15 & $22: 25$ & 23:02 & $\mathrm{X} 2.6$ & N14W08 & 10720 & 23:06:50 & Halo & 2861 \\
\hline & 2005 Jan. 16 & $21: 55$ & $22: 03$ & M2.4 & N15W19 & 10720 & & & \\
\hline & 2005 Jan. 17 & $03: 10$ & $03: 21$ & M 2.6 & N15W21 & 10720 & & & \\
\hline \multirow[t]{7}{*}{8} & 2005 Jan. 17 & 06:59 & 09:52 & $\mathrm{X} 3.8$ & N15W25 & 10720 & 09:30:05 & Halo & 2094 \\
\hline & & & & & & & 09:54:05 & Halo & 2547 \\
\hline & 2005 Jan. 18 & $15: 38$ & $15: 51$ & M 4.6 & N14W40 & 10720 & $17: 14: 20$ & 316 & 287 \\
\hline & 2005 Jan. 19 & $06: 58$ & $07: 31$ & M 6.7 & N16W53 & 10720 & 09:28:39 & 42 & 485 \\
\hline & 2005 Jan. 19 & 08:03 & $08: 22$ & X 1.3 & N15W51 & 10720 & $08: 29: 30$ & Halo & 2020 \\
\hline & & & & & & & $10: 54: 24$ & 320 & 823 \\
\hline & 2005 Jan. 19 & $10: 19$ & $10: 24$ & M 2.7 & N18W47 & 10720 & $11: 27: 31$ & 57 & 1011 \\
\hline \multirow[t]{2}{*}{9} & 2005 Jan. 20 & 06:36 & 07:01 & X7.1 & N14W61 & 10720 & 06:54:05 & Halo & 882 \\
\hline & 2005 Jan. 21 & $10: 10$ & $10: 16$ & M 1.7 & N19W81 & 10720 & $10: 58: 35$ & 292 & 273 \\
\hline \multirow[t]{7}{*}{10} & 2005 Sep. 7 & $17: 17$ & $17: 40$ & X17. & S11E77 & 10808 & & & \\
\hline & 2005 Sep. 8 & $20: 52$ & 21:06 & X5.4 & S11E74 & 10808 & & & \\
\hline & 2005 Sep. 9 & $02: 43$ & 03:00 & X1.1 & S12E68 & 10808 & & & \\
\hline & 2005 Sep. 9 & 09:42 & 09:59 & X3.6 & S11E66 & 10808 & & & \\
\hline & 2005 Sep. 9 & 19:13 & 20:04 & X6.2 & S10E58 & 10808 & 19:48:05 & Halo & 2257 \\
\hline & 2005 Sep. 10 & $16: 34$ & $16: 43$ & X1.1 & S11E47 & 10808 & & & \\
\hline & 2005 Sep. 10 & $21: 30$ & 22:11 & $\mathrm{X} 2.1$ & S13E47 & 10808 & 21:52:07 & Halo & 1893 \\
\hline \multirow[t]{4}{*}{11} & 2005 Sep. 13 & 19:19 & 19:27 & X1.5 & S09E10 & 10808 & 20:00:05 & Halo & 1866 \\
\hline & 2005 Sep. 13 & $23: 15$ & $23: 22$ & X1.7 & S10E04 & 10808 & 23:36:05 & 170 & 999 \\
\hline & 2005 Sep. 15 & 01:52 & $02: 12$ & M 1.3 & & & 03:00:06 & 200 & 424 \\
\hline & 2005 Sep. 15 & 08:30 & 08:38 & X1.1 & S12W14 & 10808 & & & \\
\hline \multirow[t]{6}{*}{12} & 2006 Dec. 5 & 10:18 & $10: 35$ & X9.0 & S07E68 & 10930 & & & \\
\hline & 2006 Dec. 6 & 01:30 & $02: 20$ & M 1.1 & S07E69 & 10930 & & & \\
\hline & 2006 Dec. 6 & 08:02 & $08: 23$ & M 6.0 & S04E63 & 10930 & & & \\
\hline & 2006 Dec. 6 & $18: 29$ & 18:47 & X6.5 & S06E63 & 10930 & & & \\
\hline & 2006 Dec. 6 & $20: 14$ & 20:19 & M 3.5 & S06E58 & 10930 & $22: 12: 04$ & 133 & 935 \\
\hline & 2006 Dec. 7 & $18: 20$ & $19: 13$ & M 2.0 & S06E45 & 10930 & $19: 37: 56$ & 128 & 830 \\
\hline \multirow[t]{2}{*}{13} & 2006 Dec. 13 & 02:14 & 02:40 & X3.4 & S06W23 & 10930 & 02:54:04 & Halo & 1774 \\
\hline & 2006 Dec. 14 & 21:07 & 22:15 & $\mathrm{X} 1.5$ & S06W46 & 10930 & 22:30:04 & Halo & 1042 \\
\hline
\end{tabular}

Notes. ${ }^{(1)}$ Bold indicates the major solar event contributing to the SPE (see text for more information). Event numbers correspond to SPEs marked in Fig. 1. 


\section{References}

Anastasiadis, A. 2002, J. Atmosph. Sol.-Terrestr. Phys., 64, 481

Bothmer, V., \& Zhukov, A. 2007, in Space Weather - Physics and Effects, ed. V. Bothmer, \& I. A. Daglis (Berlin: Springer Verlag)

Bougeret, J.-L., Kaiser, M. L., Kellogg, P. J., et al. 1995, Space Sci. Rev., 71, 231

Brueckner, G. E., Howard, R. A., Koomen, M. J., et al. 1995, Sol. Phys., 162, 357

Bühler, P., Zehnder, A., Desorgher, L., et al. 1996, in Environment modelling for Space-based Applications, ESA SP-392, 87

Cliver, E. W., Kahler, S. W., Shea, M. A., et al. 1982, ApJ, 260, 362

Daglis, I. A. 2004, Effects of space weather on technology infrastructure (Dordrecht: Kluwer Academic Publishers)

Delaboudinière, J.-P., Artzner, G. E., Brunaud, J., et al. 1995, Sol. Phys., 162, 291

Domingo, V., Fleck, B., \& Poland, A. I. 1994, Space Sci. Rev., 70, 7

Domingo, V., Fleck, B., \& Poland, A. I. 1995, Space Sci. Rev., 72, 81
Li, C., Dai, Y., Vial, J.-C., et al. 2009, A\&A, 503, 1013

Evans, H. D. R., Bühler, P., Hajdas, W., et al. 2008, Adv. Space Res., 42, 1527

Hajdas, W., Bühler, P., Eggel, C., et al. 2003, A\&A, 411, L43

Handy, B. N., Acton, L. W., Kankelborg, C. C., et al. 1999, Sol. Phys., 187, 229

Hovestadt, D., Hilchenbach, M., Bürgi, A., et al. 1995, Sol. Phys., 162, 441

Kahler, S. 1994, ApJ, 428, 837

Lin, R. P., Dennis, B. R., Hurford, G. J., et al. 2002, Sol. Phys., 210, 3

Mewaldt, R. A., Cohen, C. M. S., Labrador, A. W., et al. 2005, J. Geophys. Res., 110, A09S18

Mohammadzadeh, A., Evans, E., Nieminen, P., et al. 2003, IEEE Trans. Nucl. Sci., 50, 2272

Reames, D. V. 1999, Space Sci. Rev., 90, 413

Reames, D. V. 2009, ApJ, 693, 812

Sandberg, I., Daglis, I. A., Anastasiadis, A., et al. 2009, Space Weather, submitted

Tylka, A. J., Cohen, C. M. S., Dietrich, W. F., et al. 2005, ApJ, 625, 474 